\title{
Prevalence of Tinnitus in Patients Diagnosed with Cerebral Arteriovenous Fistula Treated with Endovascular Technique
}

\author{
Leandro José Haas ${ }^{10}$ Bruno Rafael Sabel ${ }^{20}$ Mateus Campestrini Harger ${ }^{20}$ Julia Martins ${ }^{20}$ \\ Guilherme Voltolini Staedele ${ }^{2(1)}$ Liz Caroline de Oliveira Camilo ${ }^{2}$ () Natalia Tozzi Marques ${ }^{2(0)}$ \\ ${ }^{1}$ Department of Neurosurgery and Interventional Neuroradiology, \\ Hospital Santa Isabel Blumenau, Blumenau, Santa Catarina, Brazil \\ ${ }^{2}$ Department of Health Sciences, Fundação Universidade Regional de \\ Blumenau, Blumenau, Santa Catarina, Brazil \\ Address for correspondence Bruno Rafael Sabel, physician, Fundação \\ Universidade Regional de Blumenau (FURB) Antonio da Veiga, \\ 140 Blumenau, Santa Catarina 89012-900, Brazil \\ (e-mail: brnsabel@gmail.com).
}

Int Arch Otorhinolaryngol 2022;26(3):e428-e433.

\author{
Abstract \\ Keywords \\ - vascular origin \\ tinnitus \\ - arteriovenous fistulas \\ - endovascular \\ techniques \\ - somatosounds
}

Introduction Tinnitus is characterized as the conscious and involuntary perception of sound, and it affects $\sim 30 \%$ of the population. Despite careful physical examination, the etiology of tinnitus can be established for only $30 \%$ of patients. Tinnitus is a common symptom of cerebral arteriovenous fistulas and results from increased blood flow through the dural venous sinuses, leading to turbulent arterial flow, mainly related to sigmoid and transverse sinus lesions.

Objectives To analyze the frequency of tinnitus, patient profile, and endovascular treatment characteristics in individuals diagnosed with cerebral arteriovenous fistulas. Methods A retrospective and observational study based on reviewed data from medical records on the PHILIPS Tasy system (Philips Healthcare, Cambridge, MA, USA) at the neurosurgery and interventional neuroradiology service of Hospital Santa Isabel in Blumenau-state of Santa Catarina, Brazil.

Results The profile of 68 individuals diagnosed with cerebral arteriovenous fistula who underwent endovascular treatment were analyzed. Most patients were female, aged 31 to 60 . Tinnitus affected 18 individuals. Dural fistulas were the most prevalent in the sample, and computed tomography alone was the most used diagnostic method for initial investigation.

Conclusion The prevalence of this symptom in patients diagnosed with cerebral arteriovenous fistula was found in $26.5 \%$ of this sample, mainly in women with associated comorbidities. Tinnitus remission was observed in all patients who underwent endovascular treatment to correct cerebral fistula.

\section{Introduction}

Arteriovenous fistulas are characterized as abnormal blood shunts between the arterial and venous systems without a normal capillary bed intervention. ${ }^{1}$ Most patients with dural arteriovenous fistulas (DAVFs) are in their fifth or sixth decades of life, and their symptoms vary according to the location of the lesion and the pattern of venous drainage. received

May 16, 2021

accepted after revision

September 23, 2021

published online

December 17, 2021
DOI https://doi.org/ 10.1055/s-0041-1740399. ISSN 1809-9777.

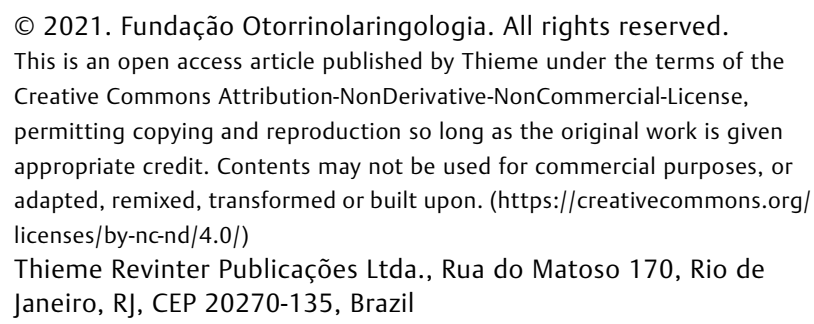


Cavernous sinus arteriovenous fistulas may cause symptoms such as ophthalmoplegia, proptosis, chemosis, retro-ocular pain, or decreased visual acuity. ${ }^{2}$ Pulsatile tinnitus is a common symptom of cerebral arteriovenous fistulas and results from increased blood flow through the dural venous sinuses, leading to turbulent arterial flow, especially when related to sigmoid and transverse sinus lesions. ${ }^{2,3}$

Tinnitus is characterized as the conscious and involuntary perception of sound, usually unwanted, in the ear, and it affects $\sim 30 \%$ of the population. ${ }^{4,5}$ The auditory perception of rhythmic noise synchronized with the heartbeat is defined as pulsatile tinnitus. ${ }^{3}$ Less than $10 \%$ of tinnitus patients suffer from pulsatile tinnitus, which is usually unilateral; however, in case of bilateral vascular pathologies, it tends to be bilateral. ${ }^{5}$ Despite careful physical examination, the etiology of vascular tinnitus can be found in only $30 \%$ of the patients, and it is better established for bilateral tinnitus. ${ }^{5}$ After headache, pulsatile tinnitus is the most common symptom of DAVFs. ${ }^{5}$ In most cases, the treatment of cerebral arteriovenous fistulas primarily involves endovascular technique and, in case of failure of the procedure, surgery and radiosurgery. In the present study, we sought to analyze the prevalence of tinnitus, patients' profiles, and endovascular treatment characteristics in individuals diagnosed with cerebral arteriovenous fistulas.

\section{Methods}

In this retrospective and observational study, data from medical records were reviewed on the PHILIPS Tasy system (Philips Healthcare, Cambridge, MA, USA) at the neurosurgery and interventional neuroradiology service of Hospital Santa Isabel, in the city of Blumenau, state of Santa Catarina, Brazil. The study was approved by the ethics committee of Hospital Alemão Oswaldo Cruz, São Paulo, under the number 29203219.2.0000.9127. The study population included 68 individuals diagnosed with cerebral arteriovenous fistula and covers the period from January 2006 to December 2017. The variables used in the study were: presence or absence of tinnitus, gender, age group, risk factors, fistula location, fistula laterality, diagnostic tests for cerebral arteriovenous fistula, as well as classification according to the type of fistula as dural, carotidocavernous, or medullary. Moreover, regarding the endovascular procedure, the type of anesthetic procedure and of embolizing material used, as well as patients' postoperative outcome were analyzed.

\section{Statistical Analysis}

The data was tabulated and analyzed on Microsoft Excel (Microsoft Corp., Redmond, WA, USA). The variables were correlated having as central point the presence of tinnitus in patients diagnosed with cerebral arteriovenous fistula. The results were compared using the non-parametric chi-square hypothesis test $\left(\mathrm{X}^{2}\right)$ to evaluate the association between qualitative variables, with a significance probability lower than $5 \%$, that is, $p<0.05$.

\section{Results}

The profile of 68 individuals diagnosed with Cerebral Arteriovenous Fistula submitted to endovascular treatment was analyzed (-Table 1). Female patients were more prevalent (61.8\%), as well as ages between 31 and 60 (53\%), and 30 individuals (44.1\%) did not have associated comorbities. In this sample, 18 patients presented with tinnitus (26.5\%). Dural fistulas were the most prevalent fistulas in the sample (47.1\%) and computed tomography (CT) alone was the diagnostic method most often used (52.9\%) for initial investigation. All individuals underwent cerebral arteriography. Only one individual (1.5\%) died (brain death) after 3 sessions, whereas $88.2 \%$ of patients had no complications from the treatment. The most often observed complication was ischemic stroke in 4 individuals (5.88\%).

- Table 2 shows data from patients with tinnitus. The analysis of this sample showed that $55.5 \%$ of the patients were female. Only one patient was under 30 years of age and the majority of patients were between 31 and 60 years old (66.6\%). About $50 \%$ of patients did not have any comorbidities associated.

The most common symptom was headache (45\%). Dural fistulas were the most prevalent in these patients (72.2\%) while a smaller proportion of patients were diagnosed with carotid-cavernous fistula (27.8\%). The diagnostic method was magnetic resonance imaging (MRI) in $55.5 \%$ of the cases. All patients underwent cerebral arteriography with digital subtraction. Complications following fistula closure had no major clinical repercussions for patients. Only one patient died. Following the endovascular treatment, after all treatment sessions had been completed, all patients exhibited improvement.

The statistical analysis was performed to establish an association between tinnitus and other variables, but the result was not significant ( $p>0.05)$.

\section{Discussion}

Benign causes of tinnitus may include impacted cerumen or otitis media, but vascular malformations can also cause tinnitus. Bilateral tinnitus has multiple etiologies, such as noise exposure, hypothyroidism, anemia, and psychological effects. When pulsatile, it is related to vascular causes, such as carotid diseases, elevated jugular bulb, and arteriovenous malformations. ${ }^{6}$ Besides headache, pulsatile tinnitus is the most common symptom of DAVFs. ${ }^{7}$

Vascular malformations such as arteriovenous fistulas, arteriovenous malformations, and cavernous hemangiomas are the most common causes of pulsatile tinnitus associated with cranial murmur. Acceleration or change in blood flow lead to audible turbulence at the site of the fistula, which decrease in intensity when the site is compressed and can be noticed by physicians during physical examination. ${ }^{7-9}$ Arteriovenous fistula symptoms appear around the sixth or seventh decades of life and affect men and women equally. ${ }^{10}$ Vascular malformations may also be associated with traumatic events, ${ }^{11}$ as described on - Table 1, which points out 
Table 1 Numerical distribution ( $\mathrm{n}$ ) and percentage (\%) of individuals by gender, age, comorbidities, symptom topography, site, laterality, diagnostic tests, number of sessions, deaths, and complications

\begin{tabular}{|c|c|c|c|}
\hline Variable & Classification & $\mathrm{n}$ & Percentage (\%) \\
\hline \multirow[t]{2}{*}{ Gender } & Male & 26 & $38.2 \%$ \\
\hline & Female & 42 & $61.8 \%$ \\
\hline \multirow[t]{3}{*}{ Age } & Under 30 & 13 & $19.1 \%$ \\
\hline & $31-60$ & 36 & $53.0 \%$ \\
\hline & Over 60 & 19 & $27.9 \%$ \\
\hline \multirow[t]{7}{*}{ Comorbidities } & Arterial Hypertension & 7 & $10.3 \%$ \\
\hline & Diabetes mellitus & 0 & $0.0 \%$ \\
\hline & Dyslipidemia & 1 & $1.5 \%$ \\
\hline & Prior trauma & 17 & $25 \%$ \\
\hline & Smoker & 3 & $4.4 \%$ \\
\hline & None & 30 & $44.1 \%$ \\
\hline & More than one & 10 & $14.7 \%$ \\
\hline \multirow[t]{2}{*}{ Topography } & Cerebral & 63 & $92.6 \%$ \\
\hline & Medullar & 5 & $7.4 \%$ \\
\hline \multicolumn{4}{|l|}{ Symptoms } \\
\hline \multirow[t]{2}{*}{ Headache } & Yes & 45 & $66.2 \%$ \\
\hline & No & 23 & $33.8 \%$ \\
\hline \multirow[t]{2}{*}{ Dizziness } & Yes & 14 & $20.6 \%$ \\
\hline & No & 54 & $79.4 \%$ \\
\hline \multirow[t]{2}{*}{ Epilepsy } & Yes & 3 & $4.4 \%$ \\
\hline & No & 65 & $95.6 \%$ \\
\hline \multirow[t]{2}{*}{ Murmur } & Yes & 14 & $20.6 \%$ \\
\hline & No & 54 & $79.4 \%$ \\
\hline \multirow[t]{2}{*}{ Proptosis } & Yes & 28 & $41.2 \%$ \\
\hline & No & 40 & $58.8 \%$ \\
\hline \multirow[t]{2}{*}{ Chemosis } & Yes & 21 & $30.9 \%$ \\
\hline & No & 47 & $69.1 \%$ \\
\hline \multirow[t]{2}{*}{ Parestesia } & Yes & 3 & $4.4 \%$ \\
\hline & No & 65 & $95.6 \%$ \\
\hline \multirow[t]{2}{*}{ Tinnitus } & Yes & 18 & $26.5 \%$ \\
\hline & No & 50 & $70.5 \%$ \\
\hline \multirow[t]{5}{*}{ Site } & $\begin{array}{l}\text { Carotid } \\
\text { Cavernous }\end{array}$ & 30 & $44.1 \%$ \\
\hline & Dural & 32 & $47.1 \%$ \\
\hline & Vertebrojugular & 2 & $2.9 \%$ \\
\hline & Dorsal & 3 & $4.4 \%$ \\
\hline & Brain stem & 1 & $1.5 \%$ \\
\hline \multirow[t]{3}{*}{ Laterality } & Right & 35 & $51.5 \%$ \\
\hline & Left & 27 & $39.7 \%$ \\
\hline & Bilateral & 6 & $8.8 \%$ \\
\hline \multirow[t]{5}{*}{ Diagnostic tests } & $\mathrm{CT}$ & 36 & $52.9 \%$ \\
\hline & MRI & 19 & $27.9 \%$ \\
\hline & $\mathrm{CT}+\mathrm{MRI}$ & 4 & $5.9 \%$ \\
\hline & Angio-CT & 1 & $1.5 \%$ \\
\hline & Other (more than one) & 8 & $11.8 \%$ \\
\hline
\end{tabular}

Table 1 (Continued)

\begin{tabular}{|l|l|l|l|}
\hline Variable & Classification & $\mathbf{n}$ & Percentage (\%) \\
\hline Number of sessions & 1 & 47 & $69.1 \%$ \\
\cline { 2 - 4 } & 2 & 18 & $26.5 \%$ \\
\cline { 2 - 4 } & 3 & 3 & $4.4 \%$ \\
\hline \multirow{3}{*}{ Death } & Yes & 1 & $1.5 \%$ \\
\cline { 2 - 5 } & No & 67 & $98.5 \%$ \\
\hline \multirow{3}{*}{ Complications } & Yes & 8 & $11.8 \%$ \\
\cline { 2 - 5 } & No & 60 & $88.2 \%$ \\
\hline Total & 68 & $100 \%$ \\
\hline
\end{tabular}

Abbreviations: Angio-CT, angiotomography; $\mathrm{CT}$, computed tomography; MRI, magnetic resonance imaging.

that $25 \%$ of patients diagnosed with arteriovenous fistulas have a previous history of trauma. In our sample, the percentage of individuals with tinnitus is $26.5 \%$, which emphasizes the high prevalence of this symptom in patients diagnosed with cerebral arteriovenous fistulas. It is also important to stress that dural fistulas were the most prevalent ones in patients presenting with this symptom (72.2\%).

Patients with tinnitus require a more accurate investigation when it is associated with recent and severe headache with progressive increase in intensity, which in the case of individuals with tinnitus must be considered a warning sign. More specifically, vascular malformations, bleeding, embolism, or ischemia should be suspected, requiring an immediate investigation of the patient. ${ }^{9}$ In the sample we analyzed, $45 \%$ of patients with tinnitus also had headache as a symptom.

Imaging studies associated with clinical investigation play an important role in diagnosing patients with tinnitus; however, no cause for tinnitus can be found in more than $30 \%$ of the patients. ${ }^{12}$ In this study, CT was the method most often used to diagnose brain fistulas (52.9\%), while MRI was the test most often used in patients with tinnitus as a symptom (55.5\%). Non-invasive imaging is widely used in diagnosis and initial patient evaluation. However, digital cerebral arteriography is the method of choice for confirming clinical suspicion of vascular malformations such as dural arteriovenous fistulas. In individuals presenting with objective tinnitus-when it can be heard also by the examinerphysicians can proceed directly to this imaging modality. ${ }^{13}$ Thus, $100 \%$ of the patients in this sample underwent the diagnostic gold standard.

Given the high prevalence and numerous etiologies of tinnitus-from self-limited and benign causes to life-threatening pathologies-physicians should conduct an in-depth evaluation of this symptom. ${ }^{9}$ Auscultation of the head, neck, and heart for cranial murmurs is an important component of the physical examination in a quiet environment with no external noises, as well as a thorough otoscopy. In addition to the above mentioned imaging tests, Doppler ultrasound of the head and neck vessels can also be used as a diagnostic tool. 


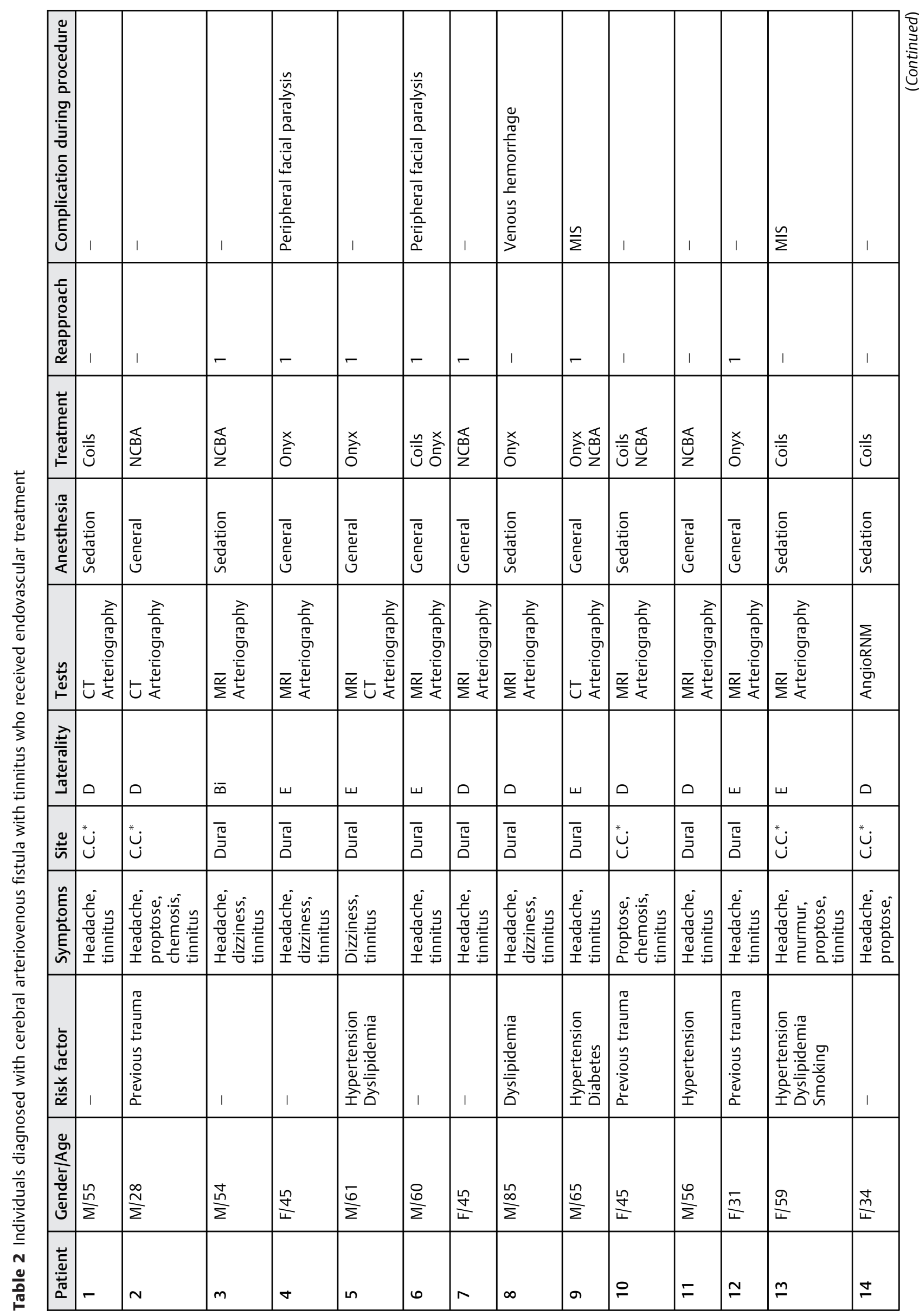




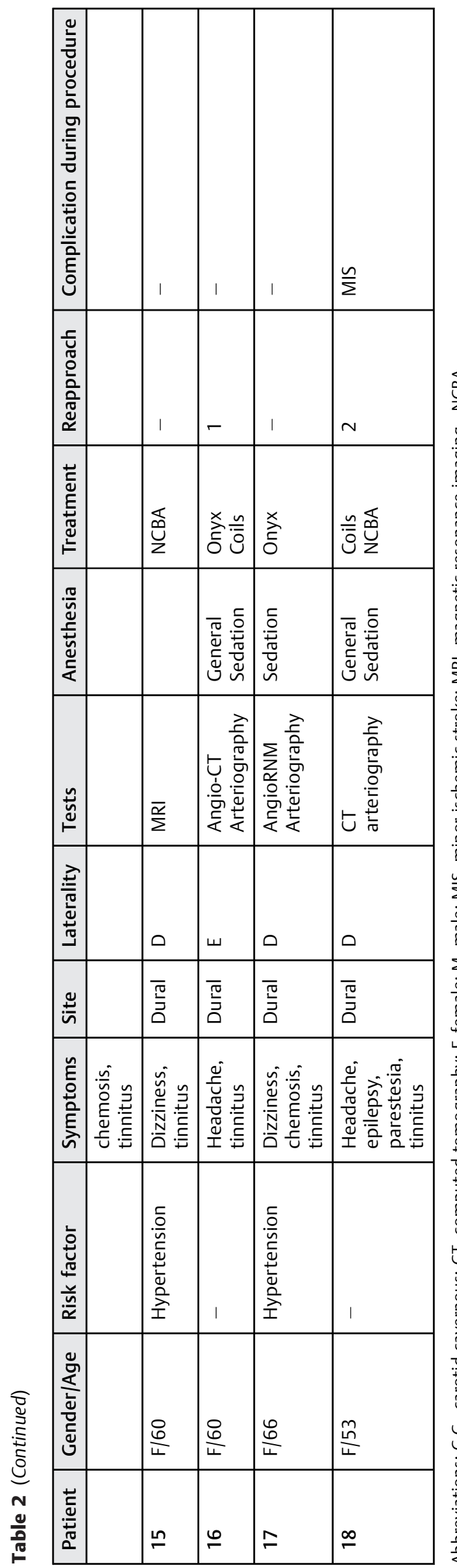

Among vascular malformations, DAVFs account for $\sim 15 \%$ of extracranial vascular malformations, and are prone to develop venous sinus thrombosis with subsequent collateral formations. ${ }^{11}$ Many patients are asymptomatic; nevertheless, they can have pulsatile tinnitus, retro-orbital pain, and cranial nerves involvement. ${ }^{14}$ In the studied sample, some individuals with tinnitus had, at the same time, proptosis, chemosis, or even paresthesia.

Intracranial hemorrhage is the most serious complication in patients with DAVFs and accounts for a mortality rate of $\sim$ $20 \%{ }^{7}$ Most patients with arteriovenous fistulas are diagnosed after a long investigation process. The risk of damage from these vascular malformations is related with the setting of venous drainage anatomy. Thus, it should be established whether, associated with tinnitus, neurological complications such as focal deficits, increased intracranial pressure, or hemorrhages may arise. ${ }^{15}$ The direction of venous drainage, such as a retrograde drainage into cortical veins, is associated with a higher risk of hemorrhage, and it is also a risk predictor. Male patients and symptomatic lesions are associated with a higher risk of hemorrhage. ${ }^{10,11}$

Direct arteriovenous fistulas stem from damage to major arteries supplying the brain. The classic cause of this damage is the formation of carotidocavernous fistulas following skull base fractures. In the sample analyzed, carotidocavernous fistulas were found in $27.7 \%$ of the tinnitus patients. ${ }^{5}$

It is assumed that, vascular tinnitus is a condition characterized by several etiologies, it should be investigated to establish a correlation between clinical findings and imaging; ideally, this should be conducted by a multidisciplinary team. Sismanis and other authors propose that the management of patients with vascular tinnitus must focus on the underlying cause, involving evaluation and follow-up not only by otolaryngologist (ENTs), but also by physicians from different specialties such as neurologists, neurosurgeons, and ophthalmologists. ${ }^{16}$

Thereby, in case a DAVFs or other intracranial vascular malformations are diagnosed based on imaging tests, the patient should be referred for evaluation from a neurosurgical team to consider other diagnostic and management options. Treatment options include embolization, radiosurgery, open surgical intervention, or wait-and-see strategies. ${ }^{10,11}$

\section{Conclusion}

Complaints of vascular tinnitus always require thorough and detailed evaluation. In $30 \%$ of the cases, there is an underlying cause that can be detected. Thorough anamnesis as well as imaging tests allow to establish a diagnosis as soon as possible and proceed with the appropriate treatment. The prevalence of this symptom in patients diagnosed with cerebral arteriovenous fistula was found in $26.5 \%$ of this sample, mainly in women with associated comorbidities. Regarding the correlation of tinnitus with the variables analyzed, the results were not significant, requiring further studies. Most cerebral arteriovenous fistulas that account for tinnitus can be treated by endovascular technique with very encouraging results. 


\section{Conflict of Interests}

The authors have no conflict of interests to declare.

\section{References}

1 Luciani A, Houdart E, Mounayer C, Saint Maurice JP, Merland JJ. Spontaneous closure of dural arteriovenous fistulas: report of three cases and review of the literature. AJNR Am J Neuroradiol 2001;22(05):992-996

2 Mossa-Basha M, Chen J, Gandhi D. Imaging of cerebral arteriovenous malformations and dural arteriovenous fistulas. Neurosurg Clin N Am 2012;23(01):27-42

3 Kircher ML, Standring RT, Leonetti JP. Neuroradiologic assessment of pulsatile tinnitus. Otolaryngol Head Neck Surg 2008;139(2, suppl): 144

4 Fussner LA, Harer KN, Poterucha TH. 51-year-old man with tinnitus. Mayo Clinic Proceedings Vol. 88 No. 6.Elsevier; 2013

5 Hofmann E, Behr R, Neumann-Haefelin T, Schwager K. Pulsatile tinnitus: imaging and differential diagnosis. Dtsch Arztebl Int 2013;110(26):451-458

6 Crummer RW, Hassan GA. Diagnostic approach to tinnitus. Am Fam Physician 2004;69(01):120-126

7 Sismanis A. Pulsatile tinnitus. Otolaryngol Clin North Am 2003;36 (02):389-402, viii

8 Levine RA, Nam EC, Melcher J. Somatosensory pulsatile tinnitus syndrome: somatic testing identifies a pulsatile tinnitus subtype that implicates the somatosensory system. Trends Amplif 2008; 12(03):242-253
9 Ridder DD. Pulsatile tinnitus. Textbook of Tinnitus New York: Springer; 467-4732010

10 Chaichana KL, Coon AL, Tamargo RJ, Huang J. Dural arteriovenous fistulas: epidemiology and clinical presentation. Neurosurg Clin N Am 2012;23(01):7-13

11 Gomez J, Amin AG, Gregg L, Gailloud P. Classification schemes of cranial dural arteriovenous fistulas. Neurosurg Clin N Am 2012; 23(01):55-62

12 Mattox DE, Hudgins P. Algorithm for evaluation of pulsatile tinnitus. Acta Otolaryngol 2008;128(04):427-431

13 Shin EJ, Lalwani AK, Dowd CF. Role of angiography in the evaluation of patients with pulsatile tinnitus. Laryngoscope 2000;110 (11):1916-1920

14 Ogilvy CS, Stieg PE, Awad I, et al;Special Writing Group of the Stroke Council, American Stroke Association. AHA Scientific Statement: Recommendations for the management of intracranial arteriovenous malformations: a statement for healthcare professionals from a special writing group of the Stroke Council, American Stroke Association. Stroke 2001;32(06): 1458-1471

15 Zipfel GJ, Shah MN, Refai D, Dacey RG Jr, Derdeyn CP. Cranial dural arteriovenous fistulas: modification of angiographic classification scales based on new natural history data. Neurosurg Focus 2009;26(05):E14

16 Sismanis A. Pulsatile tinnitus: contemporary assessment and management. Curr Opin Otolaryngol Head Neck Surg 2011;19 (05):348-357 